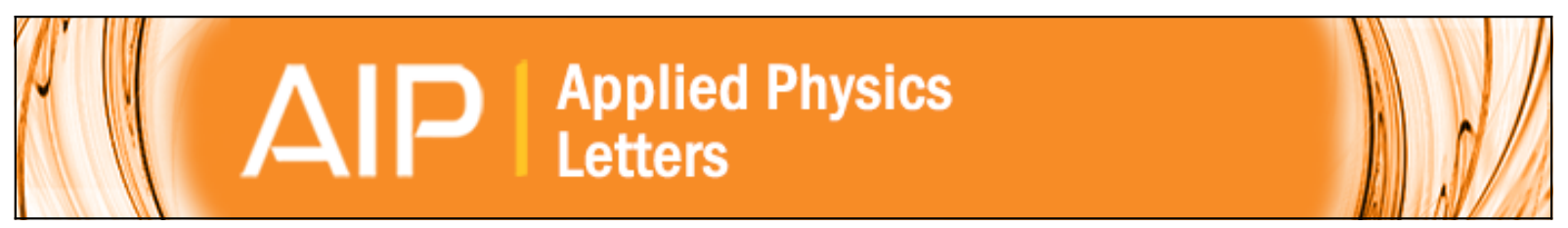

\title{
Frequency and $Q$ factor control of nanomechanical resonators
}

Johannes Rieger, Thomas Faust, Maximilian J. Seitner, Jörg P. Kotthaus, and Eva M. Weig

Citation: Applied Physics Letters 101, 103110 (2012); doi: 10.1063/1.4751351

View online: http://dx.doi.org/10.1063/1.4751351

View Table of Contents: http://scitation.aip.org/content/aip/journal/apl/101/10?ver=pdfcov

Published by the AIP Publishing

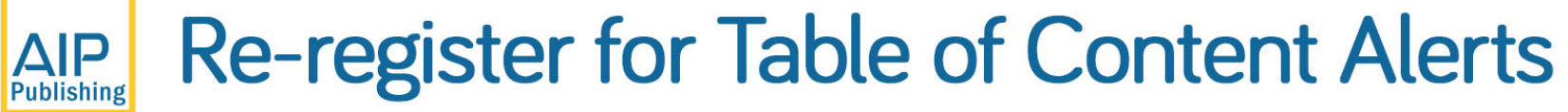

Create a profile.

Sign up today! 


\title{
Frequency and $Q$ factor control of nanomechanical resonators
}

\author{
Johannes Rieger, Thomas Faust, Maximilian J. Seitner, Jörg P. Kotthaus, \\ and Eva M. Weig ${ }^{\text {a) }}$ \\ Center for NanoScience (CeNS) and Fakultät für Physik, Ludwig-Maximilians-Universität, \\ Geschwister-Scholl-Platz 1, München 80539, Germany
}

(Received 12 July 2012; accepted 24 August 2012; published online 6 September 2012)

\begin{abstract}
We present an integrated scheme for dielectric drive and read-out of high-Q nanomechanical resonators that enable tuning of both the resonance frequency and quality factor with an applied $\mathrm{dc}$ voltage. A simple model for altering these quantities is derived, incorporating the resonator's complex electric polarizability and position in an inhomogeneous electric field, which agrees very well with experimental findings and finite element simulations. Comparing two sample geometries demonstrates that careful electrode design determines the direction of frequency tuning of flexural modes of a string resonator. Furthermore, we show that the mechanical quality factor can be voltage reduced sixfold. (C) 2012 American Institute of Physics.

[http://dx.doi.org/10.1063/1.4751351]
\end{abstract}

Control of small-scale mechanical systems is essential for their application. Resonant micro- and nanoelectromechanical systems (M/NEMS) have both proven themselves technologically viable (frequency filtering in cell phones, ${ }^{1}$ gyroscopes, ${ }^{2}$ atomic force microscope (AFM) cantilevers ${ }^{3}$ ) as well as shown great promise for next-generation sensor applications (mass sensors, ${ }^{4-6}$ resonant bio sensors, ${ }^{7}$ and ultra sensitive force sensors ${ }^{8,9}$ ). Three areas of development are central to realizing the potential of high performance resonant micro- and nanomechanics: advancement of high Q geometries and materials; improved readout schemes for mechanical motion, including compactness and integrability; and increased control of the resonant behavior of the mechanics. In the field of nanomechanics, the last years have seen the advent of high $\mathrm{Q}$ silicon nitride strings under high tensile stress. ${ }^{10,11}$ Efficient integrated drive and read-out schemes have been developed to detect the sub-nanoscale motion of small-scale resonant mechanics. ${ }^{12}$ Very good tunability of the resonance frequency can be achieved by capacitive coupling of the nanomechanical element to a side electrode. ${ }^{13}$ However, the required metalization of the resonant structure reduces the room temperature quality factor significantly ${ }^{14}$ via Ohmic losses. In our lab, an efficient, room-temperature microwave mixing scheme has been developed for readout ${ }^{15}$ as well as a dielectric drive mechanism to actuate mechanics regardless of their material makeup ${ }^{16}$ importantly obviating the necessity to metallize otherwise low-loss dielectrics.

Here, we present a continuation of this development that enables tuning of both the frequency and quality factor of nanomechanical resonators in the context of this highly applicable and integrable scheme. ${ }^{15}$ Using the combined dielectric actuation and microwave readout schemes, we theoretically develop the means to controllably raise and lower the resonant frequency of various flexural modes of our mechanics as well as to broaden the mechanical resonance linewidth. This represents a scheme for $\mathrm{Q}$ factor control, ${ }^{17-19}$

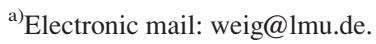

a technique widely used in AFM measurements to increase scan speed by decreasing the mechanical response time..$^{20,21}$ The theoretical relationship between the design of the electrodes and the resulting control of a given mode is validated both by experiment and simulation.

Our system is depicted in Fig. 1. A nanomechanical silicon nitride string is situated between a pair of near-lying electrodes (Fig. 1(a)). They are used to dielectrically actuate the mechanical resonance ${ }^{16}$ as well as to couple the mechanical resonator to an external microwave cavity. An equivalent circuit diagram is shown in Fig. 1(b). Deflection of the string translates into a change of the capacitance $\mathrm{C}_{\mathrm{m}}(\mathrm{t})$ between the two electrodes and thereby modulates the cavity
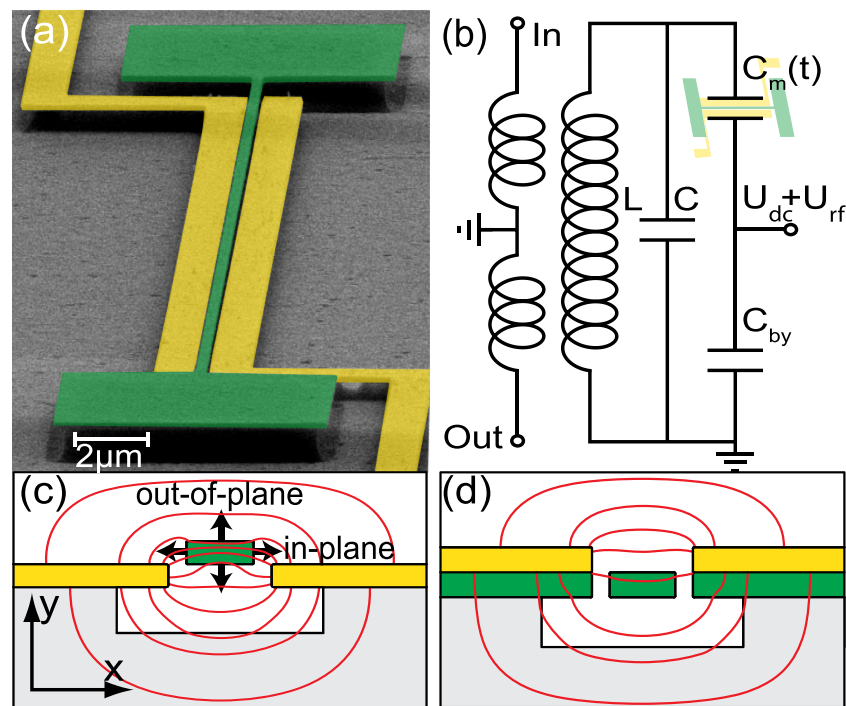

FIG. 1. (a) SEM micrograph of a $55 \mu \mathrm{m}$ long silicon nitride resonator in the configuration depicted in (c). (b) Equivalent circuit diagram of the transduction scheme with an inductively coupled microwave cavity - represented by the capacitance $\mathrm{C}$ and inductance $\mathrm{L}-$ for dielectric readout. $\mathrm{C}_{\mathrm{m}}(\mathrm{t})$ is the capacitance of the gold electrodes which is modulated by resonator displacement. The microwave bypass capacitor $\mathrm{C}_{\mathrm{by}}$ allows the additional application of a dc and rf voltage. (c) and (d) Schematic cross section with simulated field lines for the elevated and lowered geometry. The arrows in (c) describe the directions of the in-plane and out-of-plane oscillation. 
transmission signal. The mechanical oscillation can then be detected by demodulating this signal. ${ }^{15}$ To enable direct actuation of the mechanical resonator, we introduce a microwave bypass between ground and one of the electrodes using the single layer capacitor (SLC) ${ }^{22} \mathrm{C}_{\mathrm{by}}$. Thus, a dc and $\mathrm{rf}$ voltage can be applied to this electrode, whereas the other electrode is grounded via the microstrip cavity (compare Fig. 1(b)).

For this study, two sample geometries for obtaining optimized gradient field coupling are fabricated from high-stress silicon nitride films deposited on fused silica. The geometries are schematically shown in Figs. 1(c) and 1(d). Referring to the string's position with respect to the electrodes, the two structures will from now on be referenced as "elevated" (Fig. 1(c)) and "lowered" (Fig. 1(d)). The centerpiece of each structure is the $55 \mu \mathrm{m}$ long silicon nitride string resonator with a rectangular cross section of width $260 \mathrm{~nm}$ and height $100 \mathrm{~nm}$. The freely suspended resonator is bordered by two vertically offset gold electrodes, one of which is connected to the microstrip cavity with a resonance frequency of $3.5 \mathrm{GHz}$ and a quality factor of 70, while the other electrode leads to the SLC. The essential difference between the geometries is the vertical positioning of the string with respect to the gold electrodes. This affects the dielectric environment and thereby the electric field lines as depicted in Figs. 1(c) and 1(d). The simulated electric field lines for both geometries are obtained from finite element simulations using COMSOL MULTIPHYSICS and allow us to extract the electric field along the $\mathrm{x}$ - and $\mathrm{y}$-direction. These inhomogeneous electric fields cause force gradients for the inand out-of-plane modes of the resonator. They thus alter the restoring force of the respective mode and thereby its resonance frequency. ${ }^{16}$ At the same time, the mechanical quality factor can be altered with the dc voltage, as the strong electric field and high field gradient lead to velocity-dependent dielectric losses in the string material. This frequency and linewidth tuning can be described by a simple model, which agrees very well with our experimental findings and finite element simulations. The resonance frequency can be tuned over $5 \%$ and the resonance linewidth can be increased by a factor of six for a $\mathrm{dc}$ voltage of $10 \mathrm{~V}$.

We find the force gradient to be proportional to the square of the voltage and thus expect a quadratic dependence of the resonator resonance frequency on the applied dc voltage. This can be derived from the energy of the induced dipolar moment $\vec{p}$ of the dielectric resonator in an external electric field $\vec{E}$. Using a scalar, complex polarizability $\alpha=$ $\alpha^{\prime}+i \alpha^{\prime \prime}$ and introducing a dependence of the electric field on the variable coordinate $\xi$, the energy $W$ reads

$$
W=\vec{p} \cdot \vec{E}=p E=\alpha E^{2}=(E(\xi))^{2}\left(\alpha^{\prime}+i \alpha^{\prime \prime}\right) .
$$

Here $\xi$ can be the x- or y-coordinate (compare Fig. 1(c)), so the following considerations apply to both the in- and out-ofplane mode. Assuming $E(\xi)=E_{0}+E_{1} \xi$ for small displacements, the total energy can be separated into a real (stored) and an imaginary (dissipative) part

$$
\begin{gathered}
W_{\text {stored }}=\alpha^{\prime}\left(E_{0}^{2}+2 E_{0} E_{1} \xi+E_{1}^{2} \xi^{2}\right), \\
W_{\text {loss }}=\alpha^{\prime \prime}\left(E_{0}^{2}+2 E_{0} E_{1} \xi+E_{1}^{2} \xi^{2}\right) .
\end{gathered}
$$

The second derivative of the stored energy provides an additional force gradient, i.e. an electrically induced spring constant $k_{e}$

$$
k_{e}=-\frac{\partial F_{e}}{\partial \xi}=\frac{\partial^{2} W_{\text {stored }}}{\partial \xi^{2}}=\alpha^{\prime} E_{1}^{2}
$$

The shift in resonance frequency caused by $k_{e}$ can be expressed as

$$
f=\sqrt{\frac{k_{0}+k_{e}}{m}} \approx f_{0}+\frac{k_{e}}{2 m f_{0}}=f_{0}+\frac{\beta^{2} U_{\mathrm{dc}}^{2} \alpha^{\prime}}{2 m f_{0}},
$$

with a geometry-dependent proportionality between applied voltage and field gradient $E_{1}=\beta U_{\mathrm{dc}}$. Moreover, as predicted by our finite-element simulations, the sign of the gradient depends on the chosen geometry such that the out-of-plane mode changes its tuning direction between the elevated and the lowered design, which does not occur for the in-plane mode.

The quadratic tuning behavior with dc voltage is found to agree very well with the experimental data, as displayed in Fig. 2. All measurements are conducted at room temperature and a pressure of $10^{-4}$ mbars. For each mode and geometry, the mechanical spectrum is taken for different dc

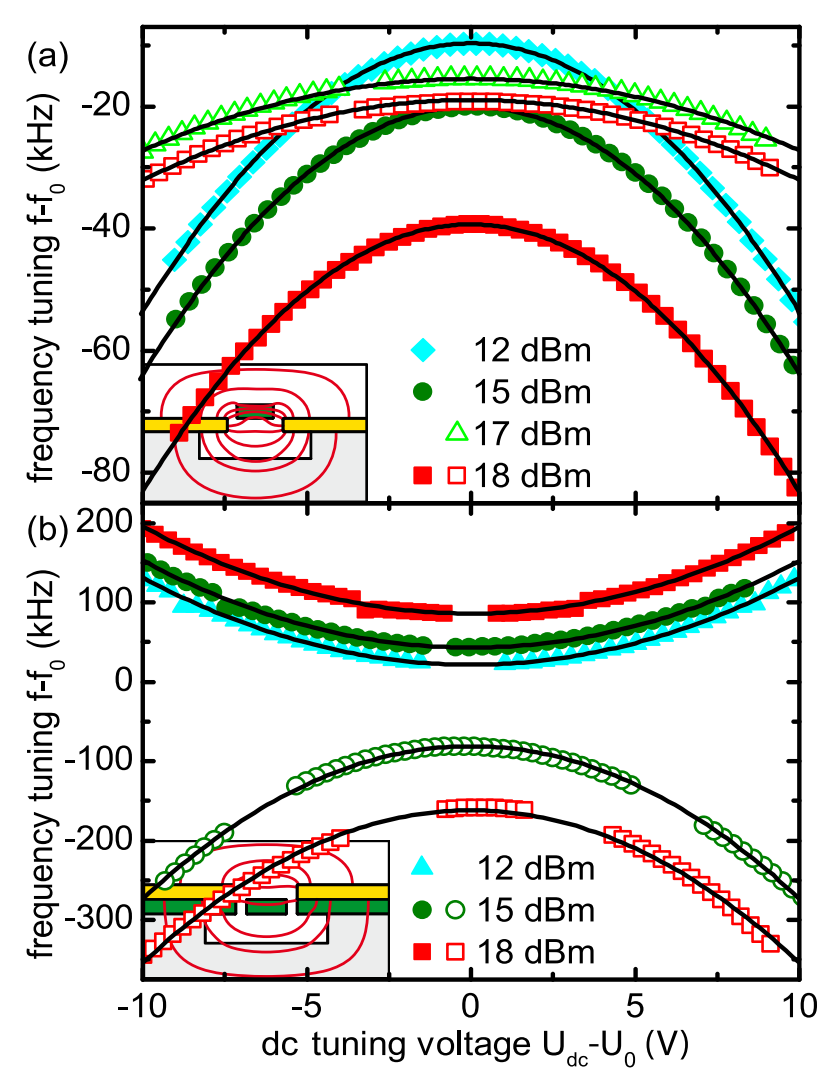

FIG. 2. Quadratic tuning of the mechanical resonance frequency with dc voltage $U_{\mathrm{dc}}$ for the two different geometries. The graphs show the deviation of the resonance frequency $f$ from the natural resonance frequency $f_{0}$ of the resonator's respective mode (in- or out-of-plane, depicted as open and filled symbols) for different microwave cavity pump powers (in $\mathrm{dBm}$ ). The solid lines are a fit of the model. (a) The force gradient has the same parity for the in-plane- as well as the out-of-plane mode. (b) With increasing $\left|U_{\mathrm{dc}}-U_{0}\right|$, the out-of-plane mode tunes upwards and the in-plane-mode downwards in frequency. 
voltages and microwave powers. The driving voltage $U_{\mathrm{rf}}$ is kept constant in every measurement. The values for $U_{\mathrm{rf}}$ lie within $80 \mu \mathrm{V}$ and $1 \mathrm{mV}$ depending on the particular mode and geometry.

A Lorentzian fit to each mechanical spectrum yields the resonance frequency and the quality factor for each parameter set. The resonance frequencies lie around $6.5 \mathrm{MHz}$ and the highest quality factor is 340000 for the out-of-plane mode in the elevated design. Note that the tuning with microwave power is a result of the effective microwave voltage ${ }^{15}$ and so is analogous to the tuning with a dc voltage. Subsequently, we fit $f=f_{0}+c_{\mathrm{dc}}\left(U_{\mathrm{dc}}-U_{0}\right)^{2}+c_{\mathrm{mw}} U_{\mathrm{mw}}^{2}$ to the tuning curves shown in Fig. 2, using the natural resonance frequency $f_{0}$ and two tuning parameters for the dc voltage and the effective microwave voltage, as the dc and high frequency polarizability might differ. We also introduce the dc offset $U_{0}$ to account for a shift (typically less than $1 \mathrm{~V}$ ) of the vertex of the tuning parabola, which is most likely caused by trapped charges in the dielectric resonator material. As the influence of the microwave field on static dipoles averages out, there is no such shift resulting from the microwave voltage $U_{\mathrm{mw}}$. Consequently, we can extract the tuning parameters for each geometry and oscillation direction. With increasing voltage $U_{\mathrm{dc}}$ and for the elevated geometry depicted in Fig. 2(a), both the in- and out-of-plane mode tune to lower frequencies, whereas for the lowered design (Fig. 2(b)), the out-of-plane mode tunes to higher frequencies, as predicted by our simulations. The solid black lines in Fig. 2 show the fit of our model with a single set of parameters for each mode in excellent agreement with the data. In the case of opposite frequency tuning, the initial frequency difference of the in- and out-of-plane modes can be evenedout, which leads to an avoided crossing caused by a coupling between the modes. ${ }^{23}$ As the data points in this coupling region deviate from normal tuning behavior, they have been omitted in Fig. 2(b).

Altering the dc or effective microwave voltage does not only shift the resonance frequency, but also influences the damping $\Gamma=2 \pi f / Q=2 \pi \Delta f$ of the mechanical resonance and thereby the measured linewidth $\Delta f$ by adding a dielectric damping contribution $\Gamma_{e}$. The dielectrically induced damping $\Gamma_{e}$ also varies quadratically with increasing voltage. This can be understood by analyzing the dissipated energy $W_{\text {loss }}$ given by Eq. (3): A time average of this quantity over one period of mechanical vibration $\xi(t)=\xi_{0} \cos (\omega t)$ gives

$$
\overline{W_{\text {loss }}}=\frac{1}{T} \int_{0}^{T} W_{\text {loss }}(\xi(t)) d t=\frac{1}{2} \alpha^{\prime \prime} E_{1}^{2} \xi_{o}^{2} .
$$

Here, we omit the $E_{0}^{2}$ term (as $\alpha^{\prime \prime}(\omega=0)=0$, otherwise static electric fields would lead to dissipation). As the mechanical stored energy $W_{\text {mech }}=\frac{1}{2} m \omega_{0}^{2} \xi_{0}^{2}$ is much larger than the electrical energy $W_{\text {stored }}$, one can approximate the additional electrical damping to be

$$
\Gamma_{e}\left(U_{\mathrm{dc}}\right)=\frac{\overline{W_{\mathrm{loss}}} \omega_{0}}{2 \pi W_{\text {mech }}}=\frac{\beta^{2} U_{\mathrm{dc}}^{2} \alpha^{\prime \prime}}{2 \pi m \omega_{0}} .
$$

The measured damping versus dc voltage is shown in Fig. 3. It displays the quadratic behavior of the damping constant

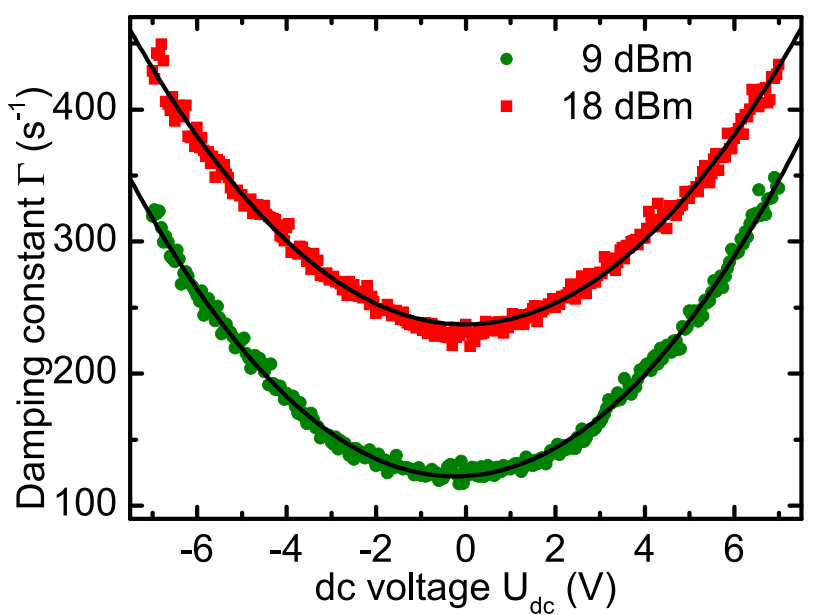

FIG. 3. Damping constant versus dc voltage for two different microwave powers, exhibiting a quadratic behavior. The solid lines are a fit of the model.

$\Gamma=\Gamma_{0}+\Gamma_{e}\left(U_{\mathrm{dc}}\right)=\Gamma_{0}+c_{\Gamma} U_{\mathrm{dc}}^{2}$ of the out-of-plane mode in the elevated design for two different microwave powers. Here, $\Gamma_{0}$ is the intrinsic damping of the resonator ${ }^{11}$ and $\Gamma_{e}\left(U_{\mathrm{dc}}\right)$ is given by Eq. (7). Again, the vertical offset between the two curves is explained by the effective microwave voltage acting analogously to a dc voltage. The solid lines in Fig. 3 are a fit of the model to the data, from which the curvature $c_{\Gamma}$ can be extracted.

Using this curvature and Eq. (7), the imaginary part of the polarizability can be expressed as $\alpha^{\prime \prime}=2 \pi c_{\Gamma} m \omega_{0} / \beta^{2}$. Similarly, employing the curvature $c_{\mathrm{dc}}$ of the parabolic frequency shift and using Eq. (5), the real part $\alpha^{\prime}$ reads $\alpha^{\prime}=2 c_{\mathrm{dc}} m f_{0} / \beta^{2}$. The ratio $\alpha^{\prime \prime} / \alpha^{\prime}=\tan (\phi)=c_{\Gamma} / 2 c_{\mathrm{dc}}$ is then independent of all resonator parameters and can be determined from the two curvatures. The measured values for damping and tuning curvatures are $c_{\Gamma}=5.2 \frac{1}{\mathrm{~V}^{2} \mathrm{~s}}$ and $c_{\mathrm{dc}}=438 \frac{\mathrm{Hz}}{\mathrm{V}^{2}}$, leading to $\tan (\phi)=0.037$. By using the Clausius-Mossotti-Relation to first calculate the (lossless) $\alpha$ using $\epsilon=7.5$, one can determine the dielectric loss tangent to be $\tan (\delta)=\epsilon^{\prime \prime} / \epsilon^{\prime}=0.016$, a value well within the range of loss tangents reported for silicon nitride thin films. ${ }^{24}$ Note that the time-varying capacitance $C_{m}(t)$ induces a dissipative current in the electrodes, which also leads to a quadratically increasing damping. ${ }^{13}$ However, using values obtained from FEM simulations for the electrode capacitance and its variation with string deflection, ${ }^{16}$ we estimate that this damping is three orders of magnitude smaller than that caused by dielectric losses. The relevant effect for the additional damping with increasing dc voltage is thus the dissipative reorientation of the dipoles in the resonator caused by its motion in a static, inhomogeneous electric field described by Eq. (6).

The dc voltage dependence of the mechanical damping $\Gamma$ was also measured at zero microwave power using an optical detection technique. ${ }^{25}$ The resulting $\Gamma_{0}$ was within a few percent of the value extracted from the $9 \mathrm{dBm}$ curve in Fig. 3 , demonstrating that a measurement at low microwave powers induces only negligible additional damping to the mechanical resonator.

In conclusion, we show dielectric frequency tuning of over $5 \%$ of the natural resonance frequency for nanomechanical resonators in an all-integrated setup that requires no metallization of the resonant mechanical structure itself. This 
scheme thus maintains an excellent quality factor of up to 340000 at $6.5 \mathrm{MHz}$ and $300 \mathrm{~K}$. Furthermore, by careful design of the geometry, one can choose the tuning behavior of the out-of-plane mode to be either upward or downward in frequency and thus tune the two orthogonal resonator modes both in the same or in opposite directions. We demonstrate that dielectric losses become highly relevant when using nanoscale electrode geometries generating large field gradients providing high tunability. This allows to directly measure the ratio of the real and the imaginary part of the resonator's polarizability by monitoring the mechanical resonance. The resulting loss tangent agrees very well with material properties of silicon nitride. We demonstrate that the dielectric losses cause additional damping of the mechanical resonance, which increases quadratically with the applied dc bias. This could be used as a $\mathrm{Q}$ factor control ${ }^{17,18,26}$ that does not require any active electronics such as a phase-locked loop but rather a single dc voltage. Such a $\mathrm{Q}$ factor control can be employed to increase the bandwidth of NEMS sensors significantly, leading to much more adaptable devices. Without the need for active electronics, this could prove to be very well suited for integrated designs. A full-fledged $Q$ factor control however requires the possibility to also increase the quality factor. A possible realization - again without the need for external, active feedback - is the backaction caused by the read-out microwave cavity. This allows to reduce the mechanical resonance linewidth and even enter the regime of self-oscillation. ${ }^{15}$ Backaction can also be used to broaden the linewidth, but we find the effect of dielectric losses to be more pronounced in our setup (a factor of six in linewidth broadening rather than a factor of two). Thus, together with microwave cavity backaction the mechanical resonance linewidth can be controlled from a few $\mathrm{Hz}$ up to more than $100 \mathrm{~Hz}$, thereby tuning the mechanical bandwidth by about two orders of magnitude. Finally, we imagine that the scheme presented can also be employed to build self-sensing AFM cantilevers ${ }^{27}$ with tunable bandwidth and resonance frequency that are not subject to the bandwidth limitations of the normally employed piezo drive and could thus be used in multifrequency force microscopy schemes. ${ }^{28}$

Financial support by the Deutsche Forschungsgemeinschaft via Project No. Ko 416/18, the German Excellence Initiative via the Nanosystems Initiative Munich (NIM) and
LMUexcellent, as well as the European Commission under the FET-Open project QNEMS (233992) is gratefully acknowledged. We thank Darren R. Southworth for critically reading the manuscript.

${ }^{1} \mathrm{~J}$. Bouchaud and H. Wicht, in Society of Photo-Optical Instrumentation Engineers (SPIE) Conference Series, edited by A. K. Henning (2005), Vol. 5717, pp. 50-54.

${ }^{2}$ R. Dean and A. Luque, IEEE Trans. Ind. Electron. 56, 913 (2009).

${ }^{3}$ G. Binnig, C. F. Quate, and C. Gerber, Phys. Rev. Lett. 56, 930 (1986).

${ }^{4}$ A. K. Naik, M. S. Hanay, W. K. Hiebert, X. L. Feng, and M. L. Roukes, Nat. Nanotechnol. 4, 445 (2009).

${ }^{5}$ M. Li, E. B. Myers, H. X. Tang, S. J. Aldridge, H. C. McCaig, J. J. Whiting, R. J. Simonson, N. S. Lewis, and M. L. Roukes, Nano Lett. 10, 3899 (2010).

${ }^{6}$ J. Chaste, A. Eichler, J. Moser, G. Ceballos, R. Rurali, and A. Bachtold, Nat. Nanotechnol. 7, 301 (2012).

${ }^{7}$ T. P. Burg, M. Godin, S. M. Knudsen, W. Shen, G. Carlson, J. S. Foster, K. Babcock, and S. R. Manalis, Nature 446, 1066 (2007).

${ }^{8}$ H. J. Mamin and D. Rugar, Appl. Phys. Lett. 79, 3358 (2001).

${ }^{9}$ C. A. Regal, J. D. Teufel, and K. W. Lehnert, Nat. Phys. 4, 555 (2008).

${ }^{10}$ S. S. Verbridge, J. M. Parpia, R. B. Reichenbach, L. M. Bellan, and H. G. Craighead, J. Appl. Phys. 99, 124304 (2006).

${ }^{11}$ Q. P. Unterreithmeier, T. Faust, and J. P. Kotthaus, Phys. Rev. Lett. 105, 027205 (2010).

${ }^{12}$ K. Ekinci, Small 1, 786 (2005).

${ }^{13}$ I. Kozinsky, H. W. C. Postma, I. Bargatin, and M. L. Roukes, Appl. Phys. Lett. 88, 253101 (2006)

${ }^{14}$ P.-L. Yu, T. P. Purdy, and C. A. Regal, Phys. Rev. Lett. 108, 083603 (2012).

${ }^{15}$ T. Faust, P. Krenn, S. Manus, J. P. Kotthaus, and E. M. Weig, Nat. Commun. 3, 728 (2012).

${ }^{16}$ Q. P. Unterreithmeier, E. M. Weig, and J. P. Kotthaus, Nature 458, 1001 (2009).

${ }^{17}$ T. Sulchek, R. Hsieh, J. D. Adams, G. G. Yaralioglu, S. C. Minne, C. F. Quate, J. P. Cleveland, A. Atalar, and D. M. Adderton, Appl. Phys. Lett. 76, 1473 (2000)

${ }^{18}$ T. R. Rodriguez and R. Garcia, Appl. Phys. Lett. 82, 4821 (2003).

${ }^{19}$ Springer Handbook of Nanotechnology, edited by B. Bhushan (Springer, 2010), p. $754 \mathrm{ff}$.

${ }^{20}$ J. Mertz, O. Marti, and J. Mlynek, Appl. Phys. Lett. 62, 2344 (1993).

${ }^{21}$ T. Sulchek, G. G. Yaralioglu, C. F. Quate, and S. C. Minne, Rev. Sci. Instrum. 73, 2928 (2002)

${ }^{22}$ Single layer capacitor, part number 500U04A182KT4S from Johanson Technology.

${ }^{23}$ T. Faust, J. Rieger, M. J. Seitner, P. Krenn, J. P. Kotthaus, and E. M. Weig, Phys. Rev. Lett. 109, 037205 (2012).

${ }^{24}$ R. Gould, Thin Solid Films 433, 309 (2003).

${ }^{25}$ T. Kouh, D. Karabacak, D. H. Kim, and K. L. Ekinci, Appl. Phys. Lett. 86, 013106 (2005).

${ }^{26}$ W. J. Venstra, H. J. R. Westra, and H. S. J. van der Zant, Appl. Phys. Lett. 99, 151904 (2011).

${ }^{27}$ M. Li, H. X. Tang, and M. L. Roukes, Nat. Nanotechnol. 2, 114 (2007).

${ }^{28}$ R. Garcia and E. T. Herruzo, Nat. Nanotechnol. 7, 217 (2012). 